www. revis tad y o. com

\title{
Innovación Organizativa en organismos de investigación ${ }^{1}$
}

Organizational innovation in research organizations

\author{
Carlos Martin-Rios ${ }^{1}$, Susana Pasamar ${ }^{2}$ y Cesar Gonzalez-Perez ${ }^{3}$ \\ ${ }^{1}$ Ecole hôtelière de Lausanne, HES-SO. University of Applied Sciences Western Switzerland \\ 1000 Lausanne 25, Switzerland. \\ ${ }^{2}$ Universidad Pablo de Olavide. Departamento de Dirección de Empresas y Marketing. \\ 41013 Sevilla, Spain \\ ${ }^{3}$ Consejo Superior de Investigaciones Científicas (CSIC) \\ Rúa San Roque, 2, 15704 Santiago de Compostela, España \\ carlos.martin.rios@ehl.ch; spasrey@upo.es; cesar.gonzalez-perez@incipit.csic.es
}

Fecha de recepción: 20-03-2016

Fecha de aceptación: 11-06-2016

\begin{abstract}
Public research organizations (hereinafter, PROs) are a type of knowledge organization with a strong emphasis on contributions by scientists. Although the value of PROs has seldom been questioned, their characteristics and functioning as well as the appropriate amount of funds to be supplied have received considerable attention in the media and from the scientific community. As in the case of other public services, PROs are subject to increasingly severe scrutiny and pressure for accountability forcing them to shift away from their traditional, bureaucratic type of organization. However, there are also limits to the restructuring of PROs guided by private sector models. Radical administrative change is not likely to occur within the boundaries of a formal scientific organization with its restricting rigidities and bureaucracies. Hence, organizational renewal of science organizations is a complex phenomenon of growing relevance. Recent developments in the study of organizational renewal have revealed the potential of organizational innovations for the design and improvement of internal processes and values. This longitudinal study of the evolution of a research center that is part of Spanish CSIC provides insight into the design and implementation of organizational innovations to drive strategic organizational shift from bureaucracy to collaborative and postbureaucracy, in an attempt to overcome external demands and promote higher organizational and financial diversification. The paper shows that innovations in work design and values may facilitate organizational change in, otherwise, highly rigid work settings. Innovation studies have traditionally been linked with technological change, usually related to manufacturing activities, and until recently, organizational innovation has received little systematic attention. This study offers insight into how certain management practices borrowed from private firms can be adopted in public research organizations, setting the base for developing a collaborative organization in science. We conclude that as contribution grows more complex, as it depends on collective endeavor to engage in more interdisciplinary undertakings, the search for alternative models of organization becomes critical for public services of this kind.
\end{abstract}

Keywords: Organizational innovation; renewal; collaborative culture; science organization, public institution

Resumen: La renovación organizativa de los organismos públicos de investigación (OPIs) es un fenómeno complejo y de creciente importancia, dada la transformación en la que se encuentra inmersa la actividad científica. Este estudio de caso longitudinal analiza el diseño e introducción de innovaciones organizativas en un OPI pertenenciente al CSIC. EI artículo ofrece evidencia sobre el potencial de ciertas innovaciones en el diseño organizativo y los valores del trabajo como agentes habilitadores del cambio en un entorno laboral rígido. Asimismo, muestra que la adaptación e introducción de determinadas prácticas de gestión tomadas de las organizaciones privadas a las circunstancias de los OPIs puede proporcionar una base sólida sobre la que desarrollar un modelo organizativo colaborativo para la ciencia.

Palabras Clave: Innovación organizativa; renovación; cultura de colaboración; organismo de investigación, instituciones públicas

\footnotetext{
${ }^{1}$ Agradecimientos: Agradecemos al equipo del Instituto por permitirnos acceder a sus instalaciones y, muy especialmente, la inestimable aportación del profesor Charles Heckscher por sus comentarios a los borradores de este trabajo.
} 


\section{Introducción}

La innovación en los modelos organizativos y de gestión refleja un modelo estratégico con el que superar la inercia y responder a los retos en el entorno social, tecnológico e institucional circundante (Camisón-Zornoza, Lapiedra-Alcami, Segarra-Cipres, y Boronet-Navarro 2004; Damanpour, Walker y Avellaneda 2009). La necesidad de adaptación al entorno es evidente no sólo en el ámbito privado, también en los servicios públicos, donde la eficacia de sus diseños organizacionales y sus procesos de trabajo tiene importantes implicaciones en la consecución de objetivos (Simpson y Powell 1999). La literatura propone avances organizativos que faciliten la transición desde la burocracia hacia la post-burocracia, o desde la primera hacia modelos más colaborativos, tanto para sector privado como sector público (Barzelay 1992; Heckscher y Martin-Rios, 2013; Hood y Peters 2004).

Ahora bien, tal y como sostienen Josserand, Teo y Clegg (2006), la transición de los servicios públicos (por ejemplo, la atención médica y educativa) hacia modelos post-burocráticos o de colaboración presenta dificultades intrínsecas, en particular las relacionadas con la resistencia a la remodelación de la estructura de la organización y los cambios en las actitudes, valores y comportamientos (Heckscher, 2007; Parker y Bradley 2000). Una alternativa a los modelos de cambio radical es la introducción de innovaciones organizativas (Damanpour 1991), definidas como la implementación de conocimiento nuevo o mejorado en las prácticas de la empresa o en la organización del lugar de trabajo y que tiene un impacto en el rendimiento de la empresa (Evangelista y Vezzani, 2010). El papel estratégico del conocimiento en el proceso de innovación destaca la importancia de los procesos de innovación no tecnológicos como el capital humano, la formación, la experiencia y la especialización (Foray, 2004). Estas innovaciones, como herramienta estratégica de mejora continua, favorecen la transición de un modelo organizativo a otro mediante acciones específicas y deliberadas (Parga, Martin-Rios y Criado, 2013). La atención que suscitan las innovaciones en el diseño organizativo no ha venido suficientemente acompañada de su estudio en el ámbito de las organizaciones científicas.

Los organismos públicos de investigación (en lo sucesivo, OPIs) constituyen un elemento esencial de los sistemas nacionales de ciencia y tecnología (Gibbons et al. 2004). Tradicionalmente, el principal cometido estos organismos de investigación financiados por el estado (tales como las instituciones de investigación sin ánimo de lucro, las agencias gubernamentales y los laboratorios) ha correspondido al ámbito de la ciencia básica (Nelson 1990), si bien los cambios institucionales recientes, relacionados con una menor financiación pública y unas exigencias mayores de resultados, están provocando un mayor interés por el desarrollo y transferencia de ciencia aplicada (Whitley 2011). Aunque el servicio y valor de estas organizaciones rara vez ha sido cuestionado, sus características, funcionamiento y financiación han recibido considerable atención en los últimos años (OCDE 2003). Entre las principales reivindicaciones está la llamada a modernizar sus estructuras organizativas y los sistemas de gestión, a lo cual algunos centros responden promoviendo sistemas alternativos a los modelos tradicionales de organización burocrática.

Este estudio profundiza en el desarrollo de nuevos modelos organizativos con los que los centros de investigación pertenecientes a los OPIs responden a las exigencias institucionales. En concreto, planteamos la siguiente pregunta de investigación, ¿qué iniciativas llevan a cabo los OPIs para reemplazar las estructuras, prácticas, valores y comportamientos del "enfoque antiguo" con nuevos sistemas de trabajo post-burocráticos y colaborativos?

El artículo se estructura como sigue. A continuación, se lleva a cabo una revisión de la literatura existente sobre OPIs e innovación, haciendo hincapié en la presión a la que habitualmente están sometidas en cuanto a renovación organizativa. Después se discuten los métodos y fuentes de datos seleccionados para el estudio longitudinal de caso, y luego se construye una historia detallada del proceso a partir del análisis de los datos cualitativos recogidos. Finalmente, se discuten los resultados y se sugieren algunas áreas para futuras investigaciones.

\section{Presión Para Innovar En Or- ganizaciones Científicas}

La importancia relativa de las organizaciones científicas es puesta de manifiesto por prácticamente cualquier indicador macroeconómico o social que se escoja. De acuerdo con la Main Science and Technology Indicators Database de la OCDE, más de 4,2 millones de investigadores en el área de la OCDE se dedicaban a I+D en 2011. La mayor parte de estos países, incluidos los EE.UU., realizan importantes inversiones en sus OPIs. No obstante, existen grandes variaciones en el reparto de la inversión en I+D y en la proporción de investigadores empleados en los sectores público y privado. En EE.UU., cuatro de cada cinco científicos están empleados en corporaciones y organismos privados, tres de cada cuatro en Japón, pero menos de 
uno de cada dos en la UE, donde los sistemas públicos de ciencia y tecnología desempeñan un papel medular y algunos países han construido un sistema público en torno a los OPIs; en España, por ejemplo, el Consejo Superior de Investigaciones Científicas (CSIC), el Instituto Nacional de Tecnología Aeroespacial (INTA) o el Instituto Geológico y Minero (IGME).

Estos organismos surgen con el cometido de proporcionar una infraestructura de investigación pública que complemente las investigaciones llevadas a cabo por otras instituciones en la esfera privada. Hoy día, si bien los estados continúan ejerciendo una influencia trascendental en la definición de estrategias, financiación y organización-estructura organizativa y administrativa-de los OPIs, la tendencia hacia una mayor liberalización económica, menor financiación pública, y una mayor presión para obtener resultados. Todo lo cual ha contribuido a disipar esta dualidad (Cruz-Castro y Sanz Menéndez, 2007; Cruz-Castro, Sanz-Menendez y Martinez, 2012).

Cada vez más OPIs se orientan a la prestación de servicios, en el sentido de ofrecer simultáneamente productos, procesos y rendimiento (Edvardsson, Gustafsson y Roos, 2005; Hill, 1977) que, en el caso de las organizaciones científicas gubernamentales se materializa en fomentar el desarrollo de la investigación básica y aplicada, facilitar la innovación industrial, y articular la transferencia de tecnología al sector privado (Albert, Granadino y Plaza, 2007; Beise y Stahl, 1999; Brooks, 1994).

Estas tendencias hacia una mayor complejidad reflejan la necesidad de vincular la generación de conocimiento científico con la difusión e incluso la aplicación de la I+D, lo cual, a su vez, tiene consecuencias de gran alcance en el modo en que los científicos realizan su trabajo (Mallon, Duberley y Cohen, 2005; Martinez, Azagra-Caro, y Maraut, 2013).

\section{Iniciativas De Innovación Organizativa}

El Manual de Oslo define la innovación como "la introducción de un nuevo, o significativamente mejorado producto (bien o servicio) de un proceso, de un nuevo método de comercialización o de un nuevo método organizativo, en las prácticas internas de la empresa, la organización del lugar de trabajo o las relaciones exteriores" (OCDE, 2005:56). A diferencia de las innovaciones de producto y proceso, la innovación organizativa (también denominada "de gestión" o "administrativa") permite a las organizaciones responder a los retos de su entorno, del mercado y de la propia gestión, mediante la creación, el desarrollo y la aplicación de nuevos métodos o prácticas organizativas que tienen un impacto beneficioso en la gestión de la organización (Birkinshaw et al, 2008; Damanpour, 2001; Hipp y Grupp, 2005; Lam, 2005). Según Birkinshaw y colegas, las innovaciones organizativas comprenden tanto la introducción de novedades en el modelo organizativo como en las diversas prácticas, procesos y técnicas de gestión del trabajo, con el fin de alterar las reglas y rutinas existentes y alcanzar los resultados planeados (2008). Por lo tanto, este tipo de iniciativas se materializan en nuevas estructuras internas, o en la introducción de novedades relativas a la gestión de los recursos humanos, o en cambios en la red de normas y valores de trabajo, o en la adopción de nuevas rutinas de trabajo que fomenten mayor productividad, colaboración y/o profesionalidad.

Las innovaciones pueden producirse por primera vez en la sociedad (new to the world) o ser innovaciones surgidas en otros entornos y que la organización asimila en sus prácticas por primera vez, bien con carácter innovador para el sector de actividad (new to the industry), o únicamente para la propia empresa (new to the firm). El alcance de cada una de las innovaciones introducidas varía en función de su novedad. Así, las innovaciones que se introducen por primera vez en la sociedad y en el área de actividad son las que, de ser exitosas, mayores repercusiones tienen.

Algunos estudios apuntan a que las innovaciones organizativas pueden servir de estímulo y factor de cambio en ambientes de trabajo rígidos, como los caracterizados por los servicios públicos (Damanpour et al, 1989; Damanpour et al, 2009). De la amplia diversidad de innovaciones organizativas presentes, un área particularmente relevante para los servicios públicos es la adopción de innovaciones en los procesos y rutinas no formales como la cultura organizativa y las actitudes, los valores y el comportamiento en el trabajo. Ciertos estudios han mostrado que los servicios y empresas públicas tienden a enfatizar el cambio de valores como motor fundamental de innovación organizativa (Barzelay, 1992; Damanpour et al, 1999; Damanpour et al, 2009; Harris y Wegg-Prosser, 2007; Parker y Bradley, 2000; Salge, 2011). Estos estudios contemplan la innovación como un proceso interactivo en el que la organización adapta la cultura dominante y los valores compartidos en relación a los procesos de trabajo y comportamientos (Kanter, 1988; Van de Ven y Poole, 2005). En este campo, el proceso de adopción y aplicación de las innovaciones debe ser interpretado y reformulado de acuerdo con el contexto de la organización y sus prioridades; éste es un proceso que a menudo involucra la participación activa de los líderes de la organización, así como de individuos y grupos clave dentro de la misma (Birkinshaw y Mol, 2006). 
Cabe esperar que los OPIs, como organizaciones sujetas a las rigideces características de los servicios públicos, hagan hincapié en iniciativas de innovación organizativa no estructural como respuesta a las exigencias del entorno de modernización. A pesar de su importancia en los procesos de adaptación y, en parte como consecuencia de lo novedoso del fenómeno, la innovación organizativa en estos servicios públicos ha recibido todavía escasa atención. El estudio que se muestra en este artículo pretende contribuir a la literatura existente al explorar las iniciativas de innovación articuladas en el proceso de organización de un centro de investigación público inmerso en un proceso de transformación de sus estrategias organizacionales y de financiación.

\section{Metodología}

El presente trabajo adopta una metodología de estudio de caso longitudinal (Van de Ven y Huber, 1990; Yin 1994) en un instituto de investigación perteneciente al CSIC. Tanto el nombre del Instituto como el de sus investigadores han sido eliminador por motivos de confidencialidad. Durante el período 2008-2011 se llevaron a cabo entrevistas en profundidad y recogida datos documentales. Se seleccionó un enfoque cualitativo por su potencial para proporcionar información de primera mano sobre el proceso de innovación.

A finales de 2008, este instituto lanzó una serie de iniciativas de innovación muy amplias, con el objetivo de articular una misión estratégica (en términos de fijación de objetivos de la organización), abordando cuestiones relativas a la estructura organizativa y, en especial, la cultura y la toma de decisiones, en un entorno tradicionalmente burocrático donde la presión hacia una mayor rendición de cuentas y eficiencia hace que la renovación organizativa era realmente compleja. Este proceso de transformación presentaba una oportunidad excepcional para observar un experimento natural de innovación y rediseño organizacional impulsado desde dentro.

\subsection{Recogida y análisis de la infor- mación}

La información se obtuvo entrevistando individuos ubicados en todos los niveles jerárquicos y áreas de trabajo. Por un lado, la información relativa a cuestiones organizativas se obtuvo principalmente de la mano de los gestores de mayor antigüedad (investigadores y técnicos liderando la iniciativa de innovación) y de los responsables de ciertas áreas de la organización (por ejemplo, el jefe del departamento de publicaciones o los responsables de proyectos en cada campo de especialización). Ellos proporcionaron información valiosa para examinar la secuencia de eventos a lo largo del periodo, determinar cómo se desarrollaron las diversas iniciativas de innovación, e identificar patrones en estos procesos. Por otro lado, la percepción de los actores organizacionales respecto a las iniciativas se obtuvo a través de entrevistas en profundidad a 15 empleados del instituto con diverso rango. Asimismo, durante los cuatro años de trabajo de campo tuvieron lugar múltiples conversaciones informales con estas y otras personas del instituto que contribuyeron a enriquecer y complementar la información del caso.

El tiempo de las entrevistas osciló aproximadamente entre 60 y 90 minutos. Las entrevistas fueron grabadas en audio digital, con permiso previo, y se hicieron transcripciones de los fragmentos que se consideraron pertinentes. Se utilizó una plantilla de entrevista semi-estructurada para guiar la investigación, aunque esta plantilla no se siguió estrictamente, ya que se pidió a los entrevistados que hablasen libremente acerca de lo que les parecía importante para ellos. Las entrevistas se realizaron en sucesivas oleadas hasta alcanzar el nivel de saturación teórica, esto es, cuando ya no aparecían nuevos conceptos ni categorías.

Además, se analizó en profundidad toda la documentación específica de la organización existente hasta la fecha, incluyendo los informes internos, las notas de comunicación a los empleados, las encuestas a empleados, el material de formación relacionado con las iniciativas de cambio, y todas las publicaciones internas que hacían referencia a las iniciativas de innovación.

El proceso de análisis de la información fue iterativo. El análisis de los datos recogidos durante las primeras etapas de la investigación guió y facilitó tanto la interpretación de los datos obtenidos en sucesivas etapas como la realización de estudios en profundidad de los temas destacados y de los problemáticos. Cada entrevista fue analizada con el fin de identificar y explorar temas, interpretaciones y percepciones relativas a las diversas iniciativas de innovación puestas en marcha. Estos datos se analizaron mediante un procedimiento de open-coding, agrupados en códigos secundarios o axiales y representados aquí como temas. De este modo la información recabada en las entrevistas sirvió para identificar los temas clave con dos propósitos. En primer lugar, un análisis inicial sirvió para crear el esquema de los eventos críticos en el proceso de innovación, ayudando a trazar la trayectoria de cambio seguida por el Instituto. Las fuentes secundarias de información, como los informes internos, fueron utilizadas para validar la línea de eventos sugerida. Un 
segundo análisis de las transcripciones de las entrevistas permitió inferir el contenido de las prácticas de innovación tal y como fueron aplicadas y la percepción de las personas involucradas en el proceso.

\section{Resultados: Eventos Críticos Del 'Innovation Pathway'}

El Consejo Superior de Investigaciones Científicas (CSIC) es un organismo de investigación financiado por el gobierno español y bajo su autoridad adminis- trativa. Colectivamente, el CSIC constituye el principal productor de I+D en España, y uno de los mayores de Europa. La Tabla 1 presenta los principales datos del CSIC durante el periodo 1985-2010. El CSIC está formalmente organizado como un conjunto de más de 130 institutos de investigación, cada uno de ellos especializado en un área particular del conocimiento. Existen institutos propios del CSIC e institutos mixtos entre el CSIC y un segundo socio, tal como una universidad o un gobierno regional. Cada instituto es relativamente libre para crear una organización interna en cuanto a departamentos, grupos de investigación o unidades diferenciadas.

Tabla 1. El panorama científico del CSIC (1985-2010)

\begin{tabular}{|c|c|c|c|c|c|c|c|}
\hline & 1985 & 1990 & 1995 & 2000 & 2005 & 2010 & $\begin{array}{l}2010-1985 \\
\text { (dif. en \%) }\end{array}$ \\
\hline \multicolumn{8}{|l|}{ Organización } \\
\hline Número de institutos & 107 & n.d. & 91 & $>100$ & 126 & 136 & $+27 \%$ \\
\hline $\begin{array}{l}\text { Investigadores } \\
\text { contratados }\end{array}$ & 1.252 & 1.777 & 1.850 & 2.145 & 2.444 & 3.165 & $+153 \%$ \\
\hline Financiación ${ }^{\star *}$ & 103.167 & 247.286 & 332.651 & 392.743 & 586.623 & 808.793 & $+684 \%$ \\
\hline \multicolumn{8}{|l|}{$\begin{array}{l}\text { Resultados investi- } \\
\text { gación }\end{array}$} \\
\hline Publicaciones* & n.d. & n.d. & n.d. & n.d. & 6.720 & 9.899 & $+147 \%$ \\
\hline $\begin{array}{l}\text { Ingresos por proyec- } \\
\text { tos }^{* *}\end{array}$ & 19.016 & n.d. & 31.487 & 54.073 & 333.522 & 452.269 & $+2.278 \%$ \\
\hline
\end{tabular}

* Artículos indexados por Thomson's Web of Knowledge y/o SCOPUS.

** Cantidades expresadas en miles de $€$.

Fuente: Memorias anuales del CSIC. http://www.csic.es/web/guest/memorias

El caso escogido para la investigación es un instituto propio (es decir, no mixto) de tamaño medio (entre los institutos que componen el CSIC) dedicado a la investigación en el ámbito de las ciencias sociales e humanidades. Es un instituto relativamente joven; sin embargo, las personas, los recursos y los proyectos que en el momento de la investigación conformaban la institución poseían una larga trayectoria como grupo, ya que provenían de un grupo de investigación anterior perteneciente asimismo al CSIC y adscrito a una universidad pública. La fundación como instituto autónomo se debió a una serie de logros: un rendimiento excepcional medido en términos de producción científica (publicaciones, proyectos de investigación concedida, asistencia a congresos, investigadores postdocto- rales acogidos, tesis doctorales defendidas, etc.); una reconocida capacidad de autogestión; buen rendimien-to en la asignación de recursos y gastos; y, por último, un crecimiento significativo en capital humano y áreas de investigación cubiertas.

La formalización del Instituto como entidad dio a los investigadores un grado de autonomía, sobre todo en la elección de su misión investigador y el modelo de organización para su consecución, que pronto cristalizó en la necesidad de formular un 'sentido', 'valor' o 'estrategia' científica de largo alcance. Dicha estrate-gia fijó como objetivo fundacional la colaboración multidisciplinar, evitando sesgos disciplinares, in-tentando reflejar la riqueza y diversidad de los campos 
académicos de interés y proporcionando herramientas y oportunidades para la investigación interdisciplinar. Se pretendía que los resultados científicos mejorasen con la creación de un espacio para la formación de alianzas de colaboración científica y aprendizaje. Para ello, se optó por albergar a especialistas de una amplia variedad de campos.

El trabajo multidisciplinar precisa de una nueva visión estratégica y también nuevas actitudes, valores y comportamientos (Jackson, 1996), que entran en conflicto con las nociones más convencionales de lo que supone el trabajo en las organizaciones burocráticas, por lo que plantea la necesidad de nuevas soluciones organizacionales. Sin embargo, las políticas públicas de investigación en España están fuertemente basadas en el concepto de áreas de conocimiento o disciplinas, y aunque la idea abstracta de la interdisciplinariedad es con frecuencia elogiada, su aplicación práctica en un entorno diseñado para institutos tradicionales, monolíticos y orientados a disciplinas específicas es ardua. La ausencia de referentes nacionales en la introducción de modelos científicos colaborativos con efectos organizativos permanentes, persuadió a los responsables del Instituto de la conveniencia de ampliar la búsqueda a un abanico más amplio de organizaciones, como las propuestas en la empresa privada (Adler y Heckscher 2013; Adler, Kwon y Heckscher 2008; Heckscher y Adler 2006; Heckscher y Martin-Rios, 2013). De este análisis se extrajo la necesidad de introducir un nuevo modelo de organización y gestión del trabajo, así como una nueva cultura organizativa que pusieran las bases de un modelo colaborativo. Ambas iniciativas de innovación desarrolladas son analizadas en detalle a continuación.

\subsection{Innovación y estructura organizati- va}

En términos de organización formal, originalmente el Instituto se organizaba en torno a los procesos estándar de la labor científica básica, que implicaban una considerable burocratización de procesos, al tiempo que una gran autonomía en la toma de decisiones por parte de los investigadores (Fernández-Esquinas et al. 2009). La estructura formal de la organización incluía descripciones detalladas de las diferentes funciones, de los canales formales de toma de decisión y de comunicación, de ciertos procedimientos estándar y de las prácticas de gestión de recursos humanos. Estas descripciones y la elevada formalidad proporcionadas por el CSIC como organización "matriz" del Instituto, contrastaban fuertemente con el predominio de mecanismos informales para la resolución de problemas dentro del Instituto. Esta situación de tension entre lo muy formal y lo poco formal es bastante habitual en los centros de investigación del CSIC, ya que tienden a funcionar de modo relativamente autónomo, al menos en la práctica, como pequeñas organizaciones; pero, al mismo tiempo, no dejan de pertenecer a una matriz institucional altamente burocratizada y con una fuerte tradición formalista. Los investigadores más veteranos eran conscientes de las consecuencias negativas que esta tensión tenía en el proceso de investigación y la producción de resultados. Esto se refleja en las siguientes palabras de un investigador:

“La coordinación se estaba haciendo imposible. Había un procedimiento oficial para todo. Sin embargo, en la medida de lo posible se evitaban por lo tedioso y engorroso. La gente no sabía qué hacer y no había ningún control".

Durante cerca de un año, se llevó a cabo una revisión de la literatura académica, así como de informes públicos y de documentación de empresas de consultoría de servicios en materia de organización. Se invitó a expertos nacionales e internacionales en diseño y transformación organizacional para obtener sus opiniones en este proceso. Se llevó a cabo una evaluación comparativa informal de lo que otros centros de I+D habían hecho anteriormente en términos de organización. Además, se dio la oportunidad a todos los empleados del instituto para que sus opiniones fuesen conocidas y tenidas en cuenta, y para que participasen en la iniciativa de cambio. La conclusión alcanzada fue que el enfoque "centrado en el líder" que conecta las líneas de investigación del centro con el campo de especialización del líder y más comúnmente utilizado, tenía mal encaje con la visión del Instituto como un campo de investigación transversal y fértil. Como el director señaló,

"A menudo, los institutos de investigación son sólo una amalgama de varios grupos de investigación independientes que trabajan para un director. Mi objetivo es que nuestro instituto sea un grupo de equipos que trabajen de forma interdependiente y dominen diferentes aspectos de investigación y que, cuando sea necesario, puedan reunirse para ofrecer una "visión única" del Instituto"

Las iniciativas de transformación arrancaron con la celebración de una serie de reuniones para discutir el futuro del instituto. Dos órganos de gobierno, el Claustro Científico y el Comité de Organización, se establecieron para diseñar y supervisar el establecimiento de una estructura formal y flexible. Finalmente, el Comité de Organización optó por una estructura matricial para proporcionar un enfoque dual simultáneo en comunidades científicas, por un lado, y proyectos de traba- 
jo por el otro. Como afirmó un científico senior: "ágil significa ser flexible, pero no a costa de prescindir de una estructura formal". Los componentes básicos de la nueva estructura organizativa incluyen los siguientes:

a. Los órganos de gobierno supervisan la matriz. Éstos incluyen los anteriormente mencionados Comité de Organización y Claustro Científico.

b. En un eje de la matriz se disponen una serie de especialidades, que corresponden a disciplinas académicas o áreas de especialización, y que se definen como comunidades científicas con un cuerpo de conocimiento y/o enfoque metodológico bien definido. Cada especialidad dispone de un coordinador.

c. En el otro eje de la matriz se disponen las operaciones de I+D, como proyectos o actividades, cada una con su líder. Proyectos y actividades se conciben como esfuerzos gestionados en el tiempo, con propósitos bien definidos, y equipos y recursos asignados. Se creó un nuevo órgano de gobierno, denominado Junta de Coordinación, que reúne a todos los coordinadores de especialidades, y cuyo objeto es la resolución en el día a día de las cuestiones relacionadas con los proyectos en curso y la solución de disputas cotidianas.

d. Dando apoyo a la matriz existen varios servicios horizontales, tales como servicios de biblioteca y archivo, administración, formación interna, incubadora de empresas, o sistemas de información, cada uno dotado de una plantilla de técnicos especialmente contratados y asistidos por investigadores de las especialidades. Cada servicio horizontal dispone de un responsable.

El cambio en la forma de trabajar sugirió una reevaluación fundamental de las funciones de todas las personas involucradas. Con el fin de facilitar la transición hacia la nueva organización, los siguientes cuatro procesos de trabajo fueron llevados a cabo. Estos procesos llamaron a la participación activa de todos los miembros del Instituto.

- $\quad$ El desarrollo continuo y el fortalecimiento de una estrategia práctica y visible.

- $\quad$ El intercambio frecuente y multimodal de información entre los proyectos.

- Procesos de resolución de conflictos, evaluación y recompensa abiertos.

Procesos de "línea de visión" para asegurar que los proyectos se mantienen alineados hacia los objetivos que son considerados de valor por las diferentes partes interesadas y los "clientes".

Al mismo tiempo se diseñaron dos mecanismos específicos de toma de decisiones sobre esta estructura organizativa. Por un lado, las decisiones tácticas a nivel de proyecto serían tomadas por los líderes de los proyectos con la mayor autonomía posible, para evitar el flujo continuo de controles y aprobaciones con la dirección que había sido habitual hasta entonces. Por otra parte, las decisiones estratégicas se tomarían por consenso dentro de los grupos designados, en especial los órganos de gobierno y, en menor medida, las especialidades. La adopción de estos mecanismos de decisión implicó una cantidad significativa de entrenamiento y reconceptualización del trabajo por parte de la mayoría de los empleados del Instituto. Dada la complejidad y sofistifación de los cambios introducidos, el proceso innovador es continuado en el tiempo (en el momento de realizar esta investigación proseguían las tareas de entrenamiento y reconceptualización).

\subsection{Nueva Cultura Organizativa de Co- laboración}

Los esfuerzos realizados para lograr un nuevo modelo organizativo dieron lugar a un renovado interés por la cultura y los valores del trabajo, buscando fomentar un equilibro entre la aportación individual y colectiva en una cultura de colaboración.

Los valores fundacionales del Instituto fueron moldeados por su fundador, profesor de universidad durante 12 años hasta su renuncia y posterior traslado al CSIC para establecer el equipo de trabajo que, con el tiempo, daría paso al Instituto. Durante años la cultura organizativa igualitarista imperante favoreció un clima relajado e informal de "agentes libres" que ofrecía ventajas para los investigadores auto-motivados, como la de permitir una fuerte autonomía disciplinar frente a casi cualquier control externo. Sin embargo, también limitaba la capacidad de organizar y llevar a cabo proyectos complejos, produciendo cierta falta de capacidad de asunción de responsabilidades y de toma de decisiones. Dicha cultura ralentizaba el sistema de trabajo y lo volvía altamente dependiente del líder, convertido con frecuencia en un cuello de botella en la cadena de toma de decisiones. En palabras de un investigador senior,

"Había gente que no podía tomar una decisión o simplemente no quería hacerlo. Esto sobreexponía a [el director]. Estas personas consultaban [al director] cada decisión y esperaban que él respondiera. Lo que es peor, a [el director] parecía no disgustarle tener que 
responder a todas estas solicitudes".

Se hizo evidente, a partir de las entrevistas que realizamos a miembros del Instituto, que una de las razones subyacentes a la promoción de una cultura de colaboración yacía en la necesidad de desarrollar la nueva lógica de control que gobernara la organización. La preocupación que subyace a esta importante innovación es la necesidad de abordar la paradoja central de control y autonomía en las organizaciones científicas públicas. Dada la complejidad inherente de la labor científica, sus sistemas formales favorecen el establecimiento de formas de gestión jerárquizados y burocráticos. Estos sistemas, por su carácter rígido, son poco apropiados en entornos de trabajo colaborativos y multidisciplinares. Como respuesta, la propia organización requiere el desarrollo de mecanismos alternativos informales. A medida que el Instituto desarrolló una infraestructura de colaboración, se hicieron necesarios nuevos valores del trabajo. El análisis de las entrevistas permitió determinar cómo los miembros del Instituto interiorizaban en su discurso tres valores esenciales relacionados con la ética colaborativa: el manejo de la incertidumbre a la hora de la toma de decisiones, una orientación compartida y la centralidad de la colaboración y las relaciones interdependientes.

La transformación en algunos de los valores del trabajo se asociaba con una mayor aceptación de nuevas formas de equipos interdisciplinares y multinivel $y$, en consecuencia, que, de algún modo, se asignara menor valor a "hacer un buen trabajo" o a "conseguir los objetivos estipulados", para asignar más valor, sin embargo, a las personas que se demuestran capaces de mirar más allá de sus funciones concretas y hacer lo que sea necesario para avanzar en el propósito común, en consonancia con los postulados delineados por Adler y Heckscher (2013).

Junto a la promoción de una ética colaborativa del trabajo, las acciones de cambio se materializaron en la definición de una misión estratégica de largo alcance para la organización. Después de deliberaciones en las que participaron todas las personas del Instituto se formuló una misión con el triple propósito de:

(a) establecer objetivos a largo plazo para la organización

(b) reorientar la atención de los investigadores desde una organización jerárquica donde las disciplinas tienen una tendencia a trabajar en "silos" hacia una investigación colaborativa e interdisciplinar

(c) establecer y definir las principales partes interesadas para fomentar una organización dinámica orienta- da al servicio.

Hubo acuerdo entre los miembros de la organización en que faltaba un sentido claro de qué o quién constituía el "cliente", "usuario" o "consumidor de servicios" del Instituto. El desarrollo de un sentido unificado y una orientación estratégica en torno a los diversos stakeholders (a saber, el mundo científico, los actores institucionales y políticos, los socios comerciales y la sociedad en general), se consideraba vital pero poco claro en la organización original. Por esta razón, se esperaba que un enfoque más cuidadoso acerca de la identificación de grupos de interés y relevancia - en otras palabras, a quién y para qué los investigadores deben prestar atención (Mitchell, Agle y Wood, 1997) - provocase la aceptación del concepto del trabajo científico como algo dinámico y estrechamente asociado con la atención directa a los interesados. En discusiones posteriores, se identificaron cuatro roles bien diferenciados que los diferentes stakeholders pueden desempeñar en relación con el Instituto:

- $\quad$ Clientes, que demandan que una operación (proyecto o actividad) se lleve a cabo, aunque pueden no beneficiarse de sus resultados, y quizá no financiarla. Por ejemplo, un Ayuntamiento puede encargar un programa de actividades de divulgación científica, con lo cual es el cliente; sin embargo, estas actividades no van dirigidas al Ayuntamiento en sí, sino a sus ciudadanos.

- $\quad$ Financiadores, que sufragan el coste de llevar a cabo la operación, aunque pueden no ser quienes la demandan o quienes usan sus resultados. Por ejemplo, las diferentes Administraciones del estado habitualmente co-financian proyectos de investigación, con lo cual son financiadores, aunque estos proyectos no se llevan a cabo a petición de dichas Administraciones ni para su beneficio propio (no son clientes ni usuarios).

- Usuarios, que se benefician directamente de los resultados de un estudio, aunque pueden no ser quienes la demanden o la financien. Por ejemplo, los ciudadanos del municipio en el primer ejemplo se benefician directamente de las actividades de divulgación científica, con lo cual son usuarios; pero no las financian directamente (no son financiadores) ni las han demandado directamente (no son clientes).

- $\quad$ Prescriptores, que establecen los requisitos a tener en cuenta durante la ejecución de un proyecto, aunque pueden no financiarlo, demandarlo ni usar sus resultados. Por ejemplo, las administraciones del estado que financian proyectos de investigación suelen, además, poner condiciones acerca de su ejecución, con lo cual son también presciptores. Un organismo 
regulador que, por ejemplo, estipule condiciones legales, éticas o de seguridad biológica para un proyecto de investigación también es un prescriptor, aunque no financie ni demande ni se beneficie de los resultados de dicho proyecto.

Esta caracterización permitió al Instituto razonar mucho mejor acerca de las operaciones que se planteaban, y evaluar su diseño y justificación con mucho mayor acierto, tal y como se verificó durante la puesta en marcha de la Junta de Coordinación en 2012-2013.

La internalización de los valores y comportamientos de la nueva cultura junto con las nuevas formas y procesos de organización habrían de servir como facilitadores del cambio. En conjunto, estas mejoras revelan la magnitud del esfuerzo llevado a cabo por esta organización científica pública en cuanto a la ampliación de la noción de "cliente" hacia nuevos usuarios de servicios, en respuesta a la creciente preocupación social acerca de los costes y el valor de los servicios públicos.

\section{Discusión}

Este estudio tiene como objetivo desentrañar las actividades de innovación puestas en marcha en un instituto de investigación con el propósito de promover un modelo de organización colaborativo con el que responder a las exigencias crecientes de excelencia y multidisciplinariedad. Las ideas que subyacen a las innovaciones realizadas son las de desarrollar los valores de colaboración y propósito compartido, la eficiencia y la rendición de cuentas mediante nuevos procesos, valores y comportamientos. El énfasis en el conocimiento y la colaboración apela a una redefinición de los roles tradicionales de los individuos en el lugar de trabajo, junto con una mayor relevancia del trabajo en equipo y los acuerdos de colaboración como capacidades competitivas fundamentales (Clegg, Harris y Höpfl, 2011; Heckscher, 1994).

El instituto de investigación analizado se sumergió en un intenso proceso de reestructuración con el doble objetivo de reformular su modelo organizativo y así superar las rigideces del modelo burocrático existente y promover un cambio de valores y comportamientos a medio plazo, apoyándose para ésto en una nueva cultura organizativa de colaboración que favorezca tanto la capacidad de innovación como el establecimiento de colaboraciones interdisciplinares. El cumplimiento de los procesos externos estipulados por la organización matriz, y que eran inexcusables, fue "encapsulada" en unos servicios horizontales o cargos bien definidos, intentando que tuviesen el menor impacto posible sobre la nueva organización interna.
Ambas iniciativas impregnaron a toda la organización, desde el personal de más alto rango hasta jóvenes recién incorporados, estudiantes de doctorado y ayudantes. Al mismo tiempo, y para asistir a la gente en este esfuerzo, el instituto formuló una misión organizacional compartida con la que ofrecer una mayor orientación al servicio y a las necesidades y demandas de los múltiples agentes involucrados, puso en marcha un programa de comunicación interno que alentase, apoyase y ayudase a la gente a participar en la toma de decisiones y realizó un importante esfuerzo de formación de todo el personal.

Las organizaciones de ciencia más innovadoras intentan adaptar sus modelos organizativos para tornar más dinámicas y orientadas al servicio. De nuestro estudio se desprende que aquellas innovaciones que se llevan a cabo de manera consensuada por los miembros de la organización, con una visión compartida de la necesidad y el alcance esperado y una planificación adecuada, son menos disruptivas y, por ello, más adecuadas para el ámbito de los servicios públicos, como los OPIs. Frente una gestión del cambio disruptiva, descendente que, con frecuencia, genera resistencias entre los miembros de la organización, la innovación organizativa estratégica permite alcanzar mejores resultados en el rediseño de las estructuras formales propias de la organización burocrática hacia modelos asentados en una mayor interdependencia y colaboración. Además, y dado que los valores y los comportamientos dependen de los roles de las personas y no de las estructuras formales, una iniciativa de innovación organizativa sugiere la necesidad de intervenir sobre la cultura, los valores y ética del trabajo para permitir liberarse de los patrones existentes y responder a las nuevas condiciones.

Hasta la fecha, la evidencia de la influencia del entorno en la innovación organizacional por parte de los servicios públicos es escasa, ya que una gran mayoría de las investigaciones realizadas se limita a analizar el impacto que las regulaciones externas, y en particular las directrices políticas, tienen sobre la predisposición de una organización para innovar, más que en su capacidad real de adoptar innovaciones organizativas (Fernández y Rainey, 2006; Kimberly y Evanisko, 1981).

Queda además fuera del alcance de este artículo la constatación de que la introducción de estas innovaciones ha reportado resultados tangibles en el corto plazo, pero podemos concluir que han sentado las bases para el desarrollo de un modelo de organización científica con el que afrontar los retos de la ciencia pública en el siglo XXI. 


\subsection{Sentando Las Bases De Una Orga- nización De Investigación Colabora- tiva}

Tradicionalmente, la investigación en los OPIs se ha basado en procedimientos reiterados de verificación del conocimiento científico con el fin de producir ciencia básica que es sometida a rigurosos métodos de comprobación teóricos y, además, al escrutinio público. Siendo esto así, la forma de organización jerárquica y burocrática ha sido adecuada para la producción, en cierta forma estandarizada, de conocimiento. Sin embargo, estas mismas organizaciones se ven abocadas cada vez más a producir conocimiento aplicado, de resultados más inmediatos y tangibles. Como expertos en sus respectivos ámbitos de investigación, los científicos tienen la obligación de juzgar la importancia o criticidad de los temas, actividades, problemas de planificación, ejecución, control y toma de decisiones relacionados con sus respectivas producciones científicas.

Un fuerte énfasis en la excelencia científica, la transferencia de tecnología y el servicio a la sociedad han provocado la aparición de nuevos centros de investigación semi-públicos que ahora conviven con los centros de investigación públicos tradicionales, para hacer cumplir las invenciones técnicas y científicas y desarrollar canales de transferencia. Como resultado, el trabajo científico es cada vez más a menudo descrito como dinámico, gestionado y comercialmente orientado. El cambio en los objetivos estratégicos ha impulsado la búsqueda de nuevos diseños de organización y la aparición de soluciones de colaboración y la profesionalización de los lugares de trabajo.

El modelo colaborativo puede representar una respuesta a la creciente necesidad de una adaptación e innovación sostenible por parte de los OPIs o cualquier otro centro de investigación. La noción de cultura de colaboración representa una desviación de las normas y valores en entornos tradicionales y burocráticos (Heckscher 2007). Esta noción se refiere al conjunto diverso de conocimientos, habilidades y experiencias aportadas por personas de diferentes ámbitos a la labor de colaboración. Asimismo, introduce el concepto de 'contribución' o aportación individual al objetivo común, como un requisito que supera los límites tradicionales de desempeño centrados en la acumulación de resultados individuales. La noción de contribución en la ciencia básica y aplicada se asocia cada vez más al esfuerzo colectivo (Jones, Wuchty y Uzzi 2008), por lo que la búsqueda de modelos de colaboración resulta fundamental para este tipo de servicios públicos.

No obstante, dada la complejidad asociada a los prin- cipios de colaboración y contribución, diversos autores han advertido de las dificultades que plantean a las organizaciones que persiguen una cultura colaborativa el alinear los intereses enfrentados entre el desempeño y la obtención de resultados individuales y la necesidad de establecer relaciones de trabajo colaborativas y valores profesionales compartidos (Clanon, 1999; Hansen y Nohria, 2004). Por consiguiente, se necesita introducir nuevas prácticas de gestión (evaluación del desempeño, retribución y sistema de promoción) en un intento de dar un mayor énfasis al propósito común y los resultados grupales.

Las instituciones de ciencia deben establecer nuevas infraestructuras que rompan con las rutinas organizacionales profundamente arraigadas. Las organizaciones de colaboración buscan la integración, el intercambio de conocimientos y la colaboración efectiva en todos los niveles. En este sentido, el esfuerzo individual se fusiona con los resultados del grupo derivados de los esfuerzos de colaboración. Por tanto, se requiere la participación activa de todos los participantes en el proceso de trabajo, que debe ir acompañada de la reorganización del lugar de trabajo, lo que se espera que sea un proceso continuo de innovación, y no ya una serie de eventos discretos.

Basándonos en nuestro caso de investigación, Ilegamos a tres conclusiones sobre el proceso mismo de innovación que ayudan a desarrollar las estructuras formales que incorporan valores de colaboración. En primer lugar, la organización necesita un "modelo" de la visión para la mejora, con especial atención a las condiciones que rodean la innovación. En nuestro caso de estudio, este modelo llegó con el desarrollo de un sentido de propósito unificado y una orientación estratégica en torno a la identificación de partes interesadas y relevantes. Este modelo facilitó la creación de un plan de implementación y el establecimiento de los pasos a seguir.

En segundo lugar, es necesario abordar los cambios en los patrones de trabajo. La organización analizada estableció como objetivo clave la sustitución de los valores y comportamientos de la vieja mentalidad "cowboy" (Gouldner, 1957) donde cada persona trabajaba de forma independiente en sus propios esfuerzos de investigación, relacionados con sus disciplinas académicas particulares, hacia un enfoque de colaboración con valores basados en la interdependencia y el compromiso en esfuerzos interdisciplinares en torno a una noción amplia de la labor científica y estrechamente asociada con la atención a todas las partes interesadas. En tercer y último lugar, todo esto implica el establecimiento de ciertas prácticas de gestión y organización del trabajo (en el caso analizado, una 
estructura matricial) que facilite la adaptación a los cambios en los principios del trabajo, la organización y los resultados. Con la creación de una vía clara de innovación, la organización marca el ritmo hacia la institucionalización basada en principios de colaboración.

\section{Conclusión}

Los OPIs están sujetos cada vez más al escrutinio público y a cierta presión para la rendición de cuentas, lo que les obliga a transformar sus estructuras organizativas así como sus infraestructuras de trabajo del mismo modo que otras instituciones de carácter privado.

En este artículo hemos destacado los pasos relacionados con la transformación de un instituto de investigación burocrático en una organización claramente post-burocrática y colaborativa. El compromiso con la cultura colaborativa puede servir como un importante factor de cambio en entornos de trabajo rígidos, ya sean públicos o privados. Basándonos en una historia detallada del proceso de innovación tomado por nuestro estudio de caso, este artículo ilustra la transformación de la organización a lo largo de su proceso de innovación organizativa hasta situarla más cerca de las demandas sociales de una mayor sostenibilidad y adaptabilidad.

Existen implicaciones prácticas tanto para directivos como para todas aquellas instituciones con deficiencias en sus diseños organizacionales y sus procesos de trabajo. Aquellas organizaciones que sean capaces de establecer cuál es su visión y qué papel juega la innovación, que aborden cambios en los patrones de trabajo hacia un modelo de colaboración con valores basados en la interdependencia y el compromiso en esfuerzos interdisciplinares y que implanten ciertas prácticas de gestión y organización del trabajo para facilitar la adaptación a los cambios, estarán marcando el camino de renovación organizativa para la consecución de objetivos relacionados con la innovación y la ciencia

\section{Bibliografía}

ADLER, P. S., y HECKSCHER, C. (2013). "Collaborative ambidextrous organizations". Universia Business Review, 40, pp. 34-51.

ADLER, P. S., HECKSCHER, C., y PRUSAK, L. (2011). "Building a collaborative enterprise". Harvard Business Review Julio-agosto, pp. 95-101.
ADLER, P. S., KWON, S., y HECKSCHER, C. (2008). "Professional work: The emergence of collaborative community". Organization Science, 19, pp. 359-376.

ALBERT, A., GRANADINO, B., y PLAZA, L. (2007). "Scientific and technological performance evaluation of the Spanish Council for Scientific Research (CSIC) in the field of biotechnology". Scientometrics, 70 , pp. $41-51$.

ALDRICH, H. (1999). Organizations evolving, London, UK: Ed. Sage.

ALVESSON, M., y WILLMOTT, H. (2002). "Producing the appropriate individual: Identity regulation as organizational control". Journal of Management Studies, 39, pp. 619-644.

ARMBRUSTER, H., BIKFALVI, A., KINKEL, S. y LAY, G. (2008). "Organizational innovation: The challenge of measuring non-technical innovation in large-scale surveys". Technovation, 28, pp. 644-657.

ARROW, K. J. (1962). "Economic welfare and the allocation of resources for invention". In: The rate and direction of inventive activity. Princeton. NJ: Princeton University Press, pp. 609-636.

BARZELAY, M. (1992). Breaking through bureaucracy: A new vision for managing in government. Berkeley, CA: University of California Press

BEISE, M., y STAHL, H. (1999). "Public research and industrial innovations in Germany". Research Policy, 28, pp. 397-422.

BIRKINSHAW, J. (2010). Reinventing management: Smarter choices for getting work done. San Francisco, CA: Ed. John Wiley \& Sons

BIRKINSHAW, J., y MOL, M. (2006). "How management innovation happens". Sloan Management Review, 47, pp. 81-88.

BIRKINSHAW, J., HAMEL, G., y MOL, M. (2008). "Management innovation". Academy of Management Review, 33, pp. 825-845.

BROOKS, H. (1994). "The relationship between science and technology". Research Policy, 23, pp. 477486.

CAMISON-ZORNOZA, C., LAPIEDRA-ALCAMI, R., SEGARRA-CIPRES, M., y BORONET-NAVARRO, M. (2004). "A meta-analysis of innovation and organizational size". Organization Studies, 25, pp. 331-361. 
CHILD, J., y GUNTHER MCGRATH, R. (2001). “Organizations unfettered: Organizational form in an information-intensive economy". Academy of Management Journal, 44, pp. 1135-1148.

CLANON, J. (1999). "Organizational transformation from the inside out: Reinventing the MIT Center for Organizational Learning". Learning Organization, 6(4), pp. 147-156.

CLEGG, S., HARRIS, M., y HÖPFL, H. (2011). Managing modernity: Beyond bureaucracy? Oxford: Oxford University Press.

CRUZ-CASTRO, L., y SANZ-MENENDEZ, L. 2007. "New legitimation models and the transformation of the public research organizational field". International Studies of Management and Organization, 37(1), pp. 27-52.

CRUZ-CASTRO, L., SANZ-MENENDEZ, L. and MARTINEZ, C. (2012), "Research centers in transition: patterns of convergence and diversity". The Journal of Technology Transfer, 37(1), pp. 18-42.

DAMANPOUR, F. (1991). "Organizational innovation: A meta-analysis of effects of determinants and moderators". Academy of Management Journal, 34, pp. 555-590.

DAMANPOUR, F., y SCHNEIDER, M. 2006. "Phases of the adoption of innovation in organizations: Effects of environment, organization and top managers". British Journal of Management, 17, pp. 215-236.

DAMANPOUR, F., y SCHNEIDER, M. (2009). "Characteristics of innovations and innovation adoption in public organizations: Assessing the role of managers". Journal of Public Administration Research and Theory, 19, pp. 495-522.

DAMANPOUR, F., SZABAT, K. A., y EVAN, W. (1989). "The relationship between types of innovation and organizational performance". Journal of Management Studies, 26, pp. 587-602.

DAMANPOUR, F., WALKER, R., y AVELLANEDA, C. (2009). "Combinative effects of innovation types and organizational performance: A longitudinal study of service organizations". Journal of Management Studies, 46, pp. 650-675.

EDVARDSSON, B., y GUSTAFSSONY ROOS, A. (2005). "Service portraits in service research: A critical review". International Journal of Service Industry Management, 16, pp. 107-121.
EVANGELISTA R., y VEZZANI, A. (2010). "The economic impact of technological and organizational innovations: A firm level-analysis". Research Policy, 39, pp. 1253-1263.

FERNANDEZ, S., y RAINEY, H. G. (2006). "Managing successful organizational change in the public sector". Public Administration Review, 66, pp. 168-176.

FERNÁNDEZ-ESQUINAS, M., MERCHÁN-HERNÁNDEZ, C., y RAMOS-VIELBA FERNÁNDEZ, I. (2010). "Key knowledge providers as sources of business innovation" Industry and Higher Education, 24(3), pp. 189-201.

FERNÁNDEZ-ESQUINAS, M., SEBASTIÁN J., LÓPEZ-FACAL, J., y TORTOSA-MARTORELL. E. (2009). "Anillos de crecimiento en el árbol de la ciencia. La evolución institucional del Consejo Superior de Investigaciones Científicas" Revista Internacional de Sociología, 67(2), pp. 251-284.

GIBBONS, M., LIMOGES, C., NOWOTNY, H., SCHWARTZMAN, S., SCOTT, P., y TROW, M. (1994). The new production of knowledge: The dynamics of science and research in contemporary societies. Thousand Oaks, CA: Ed. Sage.

GREENWOOD, R., y HININGS, C.R. (1996). "Understanding radical organizational change: Bringing together the old and the new institutionalism". Academy of Management Review, 21, pp. 1022-1054.

GOULDNER, A. W. (1957). "Cosmopolitans and locals: Toward an analysis of latent social roles". Administrative Science Quarterly, 2, pp.281-306.

HANSEN, M., y NOHRIA, N. (2004). "How to build collaborative advantage". MIT Sloan Management Review, 46, pp. 22-30.

HARRIS, M., Y WEGG-PROSSER, V. (2007). "Post-bureaucracy and the politics of forgetting: The management of change at the BBC, 1991-2002". Journal of Organizational Change Management, 20(3), pp. 290-303.

HECKSCHER, C. (1994). Defining the post-bureaucratic type. In: The post-bureaucratic organization. New York: Oxford University Press, pp. 14-62

HECKSCHER, C. (2007). The collaborative enterprise. New Haven, CT: Yale University Press.

HECKSCHER, C., y ADLER, P. (2006). The corporation as a collaborative community: Organization in 
the knowledge-based economy. Oxford University Press: Oxford

HECKSCHER, C., y MARTIN-RIOS, C. (2013). "Looking back, moving forward: Toward collaborative universities". Journal of Management Inquiry, 22(1), pp. 136-139.

HILL, T. P. (1977). On goods and services. Review of Income and Wealth, 23, pp. 315-18.

HININGS, C. R., y GREENWOOD, R. (1988). The $d y-$ namics of strategic change. Basil Blackwell: Oxford

HIPP, C., y GRUPP, H. (2005)." Innovation in the service sector: the demand for service-specific innovation measurement concepts and typologies". Research Policy, 34, pp. 517-535.

HOOD, C., y PETERS, G. (2004). "The middle aging of new public management: Into the age of paradox?" Journal of Public Administration Research and Theory, 14, pp. 267-282.

JACKSON, S. E. (1996). The consequences of diversity in multi-disciplinary work teams. In: Handbook of work group psychology. Chichester, UK: Wiley, pp. 53-76.

JONES, B., WUCHTY, S., y UZZI, B. (2008). "Multi-university research teams: Shifting impact, geography, and stratification in science". Science, 322(5905), pp. 1259-1262.

JOSSERAND, E., TEO, S., y CLEGG, S. (2006). "From bureaucratic to post-bureaucratic: the difficulties of transition". Journal of Organizational Change Management, 19(1), pp. 54-64.

KANTER, R. (1988). When a thousand flowers bloom: Structural, collective and social conditions for innovation in organization. In: Research in Organizational Behavior. Greenwich, Conn.: JAI Press, pp.169211.

KIMBERLY, J. R., y EVANISKO, J. M. (1981). "Organizational innovation: The influence of individual, organizational and contextual factors on hospital adoption of technological and administrative innovation". Academy of Management Journal, 24, pp. 689-713.

LAM, A. (2005). Organizational innovation. In: The Oxford handbook of innovations. Oxford: Oxford University Press, pp. 115-147
MALLON, M., DUBERLEY, J., y COHEN, L. (2005). "Careers in public sector science: Orientations and implications". R\&D Management, 35, pp. 395-407.

MARTINEZ, C., AZAGRA-CARO, J. y MARAUT, S. (2013). "Academic investors, scientific impact and the institutionalization of Pasteur's quadrant in Spain". Industry and Innovation, 20(5), pp. 438-455.

MCNULTY, T., y FERLIE. E. (2004). "Process transformation: Limitations to radical organizational change within public service organizations". Organization Studies, 25, pp. 1389-1412.

MITCHELL, R. K., AGLE, B., y WOOD, D. (1997). "Toward a theory of stakeholder identification and salience: Defining the principle of who and what really counts". Academy of Management Review, 22, pp. 853-886.

NELSON, R. R. (1990). "Capitalism as an engine of progress”. Research Policy, 19, pp. 193-214.

PARGA, E., MARTIN-RIOS, C., y CRIADO, F. (2013). "Organizational and management innovation as a driving force of business renewal". Journal of Technology Management \& Innovation, 8(2), pp. 132-143.

PARKER, R., y BRADLEY, L. (2000). "Organizational culture in the public sector: Evidence from six organizations". International Journal of Public Sector Management, 13(2), pp.125-141.

SALGE, T. (2011). "A behavioral model of innovative search: Evidence from public hospital services". Journal of Public Administration Research and Theory, 21(1), pp. 181-210.

SIMONS, R. (2005). Levers of organization design. Boston, MA.: Harvard Business School Press.

SIMPSON, B., y POWELL, M. (1999). "Designing research organizations for science innovation". Long Range Planning, 32, pp. 441-451.

STRAUSS A., y CORBIN, J. (1998). Basics of qualitative research. Thousand Oaks, CA: Ed. Sage.

VAN DE VEN A., y HUBER, G. P. (1990). "Longitudinal field research methods for studying the process of innovation". Organization Science, 1, pp. 213-219.

VAN DE VEN, A., y POOLE, M. S. (2005). "Alternative approaches for studying organizational change". Organization Studies, 26, pp. 1377-1404. 
WALTER, R. (2006). "Innovation type and diffusion: An empirical analysis of local government". Public Administration, 84, pp. 311-335.

WEJNERT, B. (2002). "Integrating models of diffusion of innovations: A conceptual framework". Annual Review of Sociology, 28, pp. 297-326.
WHITLEY, R. (2011). "Changing governance and authority relations in the public sciences". Minerva, 49, pp. 359-385.

YIN, R. K. (1994). Case study research: Design and methods. Newbury Park, CA: Ed. Sage. 